\title{
Mode stresses for the interaction between two inclined cracks in bonded two half planes
}

\begin{abstract}
The various mode of stresses for the interaction between two inclined cracks in the upper part of bonded two half planes which are normal stress (Mode I), shear stress (Mode II), tearing stress (Mode III) and mixed stress was studied. For this problem, the modified complex potentials (MCPs) method was used to develop the new system of hypersingular integral equations (HSIEs) by applying the conditions for continuity of resultant force and displacement functions with the unknown variable of crack opening displacement (COD) function and the right hand terms are the tractions along the crack. The curve length coordinate method and Gauss quadrature rules were used to solve numerically the obtained HSIEs to compute the stress intensity factors (SIFs) in order to determine the strength of the materials containing cracks. Numerical solutions presented the characteristic of nondimensional SIFs at the cracks tips. It is obtained that the various stresses and the elastic constants ratio are influences to the value of nondimensional SIFs at the crack tips.purposes.
\end{abstract}

Keyword: Bonded half planes; Hypersingular integral equation; Inclined crack; Modified complex potentials; Stress intensity factors 